2

\section{Dextran-Coated, ${ }^{64} \mathrm{Cu}$-Doped Iron Oxide Nanoparticles}

Department of Chemistry ${ }^{1}$, Physics ${ }^{2}$, Biomedical Engineering ${ }^{3}$, University of California Davis, California, 95616,

*corresponding author: aylouie@ucdavis.edu

USA

\title{
RECEIVED DATE
}

Running title: Microwave Synthesis of DIO/Cu

Abstract: Research into developing dual modality probes enabled for magnetic resonance imaging (MRI) and positron emission tomography (PET) has been on the rise recently due to the potential to combine the high resolution of $\mathrm{MRI}$ and the high sensitivity of PET. Current synthesis techniques for developing multimodal probes is largely hindered in part by prolonged reaction times during radioisotope incorporation - leading to a weakening of the radioactivity. Along with a time-efficient synthesis, the resulting products must fit within a critical size range (between $20-100 \mathrm{~nm}$ ) to increase blood retention time. In this work, we describe a novel, rapid, microwave-based synthesis technique to grow dextran-coated iron oxide nanoparticles doped with copper (DIO/Cu). Traditional methods for coprecipitation of dextran-coated iron oxide nanoparticles require refluxing for 2 hours and result in approximately $50 \mathrm{~nm}$ diameter particles. We demonstrate that microwave synthesis can produce $50 \mathrm{~nm}$ nanoparticles with 5 minutes of heating. We discuss the various parameters used in the microwave synthesis protocol to vary the size distribution of $\mathrm{DIO} / \mathrm{Cu}$, and demonstrate the successful incorporation of ${ }^{64} \mathrm{Cu}$ into these particles with the aim of future use for dual-mode MR/PET imaging. 

due in part to recent breakthroughs in fused instrumentation, allowing simultaneous imaging with more than one technique. For example, in the past decade PET/Computed Tomography (CT) has been one of the most rapid growth areas in clinical imaging. ${ }^{1}$ CT provides anatomical context for the PET images, which can otherwise be difficult to interpret. ${ }^{2}$ Current attention has turned to fused PET/MRI instruments, ${ }^{3}$ two modalities which had previously been presumed to be incompatible. ${ }^{4}$ The marriage of these modalities provides images that combine the high resolution, anatomical imaging strengths of $\mathrm{MRI}$, which has greater soft tissue contrast than $\mathrm{CT}$, with ability of PET to detect biomarkers at high sensitivity, and monitor bio pathways. The development of probes detectable by both modalities can be advantageous, allowing image co-registration, and mapping of biomarkers across resolution scales. Only recently have PET/MRI dual-mode probes emerged in the literature. ${ }^{3}$ Radiolabeled probes built upon iron oxide nanoparticles, a clinical MRI agent, have been studied by our lab ${ }^{5,6,7}$ and others. ${ }^{8,9}$ A common approach to generate radiolabeled nanoparticles is to attach ${ }^{64} \mathrm{Cu}$ through chelation onto the surface. ${ }^{10}$ However, the chelation and purification process is relatively slow and the stability of chelated ${ }^{64} \mathrm{Cu}$ in vivo is often uncertain. ${ }^{11}$ It would be desirable to develop a synthesis method which could rapidly produce labeled particles that are stable in vivo - avoiding the ambiguity of whether free copper or labeled probe is providing the PET signal.

Microwave heating is known to reduce reaction times and improve synthesis yield by increasing the homogeneity of heating. ${ }^{12}$ The decreased reaction time offered by a microwave approach is extremely important to the synthesis and application of a dual modality probe. Traditional iron oxide synthesis methods, such as coprecipitation, typically require a long $(2 \mathrm{~h}+)$ reflux ${ }^{6}$ which represents a significant portion of the $12.7 \mathrm{~h}$ half life of ${ }^{64} \mathrm{Cu}$ radiolabel. ${ }^{13}$ Microwave synthesis has been demonstrated in the growth of $\mathrm{Y}-\mathrm{Fe}_{2} \mathrm{O}_{3},{ }^{14} \mathrm{Au}$ and $\mathrm{Pd}$ nanoparticles, ${ }^{15}$ and uncoated iron oxide nanoparticles. ${ }^{16,}{ }^{17}$ However, for successful medical application, iron oxide particles must be coated for hydrostatic stability, protection from degradation, and biocompatibility. In this work we develop a one-step technique to synthesize dextran-coated iron oxide nanoparticles (DIO) doped with copper (DIO/Cu) and copper-64 - a PET contrast agent - using microwave assisted heating. This method incorporates a radiolabel as part of the integral structure of the iron oxide nanoparticles. By doing this 
we predict that the copper would be more stable against release from nanoparticles in vivo compared to surface chelated copper. In addition, the release of free copper may be more easily distinguished than the loss of chelated copper in that particle breakdown would be required for the release of copper doped into the iron oxide particle core, which would result in loss of MRI signal. Nonradioactive copper was used for proof-ofprinciple studies, followed by incorporation of radioactive ${ }^{64} \mathrm{Cu}$ to validate the method. Further, we vary the synthesis conditions and observe variations in particle size, and also evaluate the magnetic properties of these particles. The microwave-assisted method significantly decreases synthesis time, which reduces the loss of radioactivity, and forms a more stable particle, and also is far simpler than other alternative methods of fabricating radio-labeled nanoparticles.

\section{Experimental Section}

$$
\text { We have previously reported size-controlled syntheses of dextran coated iron oxide nanoparticles }{ }^{6} \text { and used }
$$
this platform for our DIO/Cu synthesis. Reagents were obtained from either Sigma-Aldrich or Fisher Scientific and were used directly, unless otherwise noted. A Millipore Milli-Q purifier (18.0 M $\Omega \mathrm{cm}$, Barnstead) was used to purify water.

Preparation of reduced dextran. Reduced dextran was prepared by modifying a reported method ${ }^{7,18} .10 \mathrm{~g}$ of dextran (MW/MN = 10,000 Da) was dissolved in $100 \mathrm{ml}$ of nanopure water at $25^{\circ} \mathrm{C}$, followed by the addition of $1 \mathrm{~g}$ of sodium borohydride. The mixture was stirred for 24 hours. The $\mathrm{pH}$ was then brought to $\mathrm{pH} 5$ with $6 \mathrm{~N} \mathrm{HCl}$ and the mixture was put into membrane dialysis bag with a filter size of 15,000 Da (Spectra, California, USA) for 3 days (8-10 changes of water). The product was then lyophilized and stored at $4^{\circ} \mathrm{C}$.

Synthesis of DIO and DIO/Cu nanoparticles. DIO/Cu was prepared by first degassing nanopure water by argon bubbling. $0.02 \mathrm{mmol}$ of $\mathrm{FeCl}_{3} \cdot 6 \mathrm{H}_{2} \mathrm{O}$ was combined with $0.007 \mathrm{mmol}$ of reduced dextran and dissolved in $3.7 \mathrm{ml}$ of degassed $\mathrm{H}_{2} \mathrm{O}$. This solution was stirred for 40 min under an argon blanket at $4^{\circ} \mathrm{C} . \mathrm{FeCl}_{2} \cdot 4 \mathrm{H}_{2} \mathrm{O}(0.137$ $\mathrm{mmol}$ ) and $\mathrm{CuCl}_{2}(0.0137 \mathrm{mmol})$ were subsequently prepared by dissolving each in $1.5 \mathrm{ml}$ of degassed $\mathrm{H}_{2} \mathrm{O}$ and stored at $4{ }^{\circ} \mathrm{C}$ for 20 minutes. The prepared $\mathrm{FeCl}_{2} \cdot 4 \mathrm{H}_{2} \mathrm{O}$ and $\mathrm{CuCl}_{2}$ were quickly added to the $\mathrm{FeCl}_{3} \cdot 6 \mathrm{H}_{2} \mathrm{O}$ and reduced dextran mixture, and stirred for 5 minutes. $500 \mu \mathrm{l}$ of $\mathrm{NH}_{4} \mathrm{OH}$ was then added at a rate of $0.1 \mathrm{ml}$ every 5 seconds. The mixture was then loaded into a $30 \mathrm{ml}$ Activent microwave tube and heated in the microwave 
reactor - Automated Microwave Explorer/Discover Hybrid-12 (CEM Corporation, USA) - under various times and power. The resulting nanoparticles were evaluated after microwave heating for 5,10 , and 15 minutes while maintaining a maximum power output of $300 \mathrm{~W}$. For evaluation of the effect of power level on the synthesis, reaction time was fixed at 10 minutes and power outputs set to 150, 200, 250, and 300W (300W was the maximum power setting available). The microwave system typically is closed, with an emergency release of pressure only above a maximum threshold. Dextran coated iron oxide (DIO) was synthesized using the above synthesis method in the absence of copper.

Synthesis of Cu-64 nanoparticles. Using a similar reaction scheme to the above paragraph, $0.0137 \mathrm{mmol}$ of $\mathrm{CuCl}_{2}$ was replaced with $1 \mathrm{mCi}$ of $\mathrm{Cu}^{64} \mathrm{Cl}_{2}$ and the microwave time was set to 1 minute. Purification was performed with a 10kDa 0.5 micron $15 \mathrm{ml}$ ultra centrifugation filter and washed $3 x$ with $10 \mathrm{ml}$ of Nanopure $\mathrm{H}_{2} \mathrm{O}$.

\section{Probe Characterization:}

Transmission electron microscope (TEM) images were collected on a Phillips CM-120 transmission electron microscope operating at $80 \mathrm{keV}$. A minimum of 500 particles were measured to determine particle diameter. Dynamic light scattering (DLS) was used to determine the average hydrodynamic particle size in solution with a Nanotrac 150 particle size analyzer (Microtrac, Inc., Montgomeryville, PA) using a 150s scan time fitted with a residual less geometric eight-root regression. Elemental analysis for copper and iron was carried out with a Varian AA 220FS operated under a flow of air/acetylene and a copper lamp set to $248.3 \mathrm{~nm}$ and 324.8 $\mathrm{nm}$ for iron and copper, respectively. Relaxivity values were measured using a Bruker Minispec mq60 relaxometer operating at $1.4 \mathrm{~T}$, and $37^{\circ} \mathrm{C}$. Dextran coating was verified by infrared (IR) spectroscopy performed on a Shimadzu IR Prestige 21 Spectrophotometer using diffuse diffraction with $\mathrm{KBr}$ as the dilutant. Two tailed student $T$ tests with equal variances of $n=3$ was performed to test the significance between $D I O / C u$ points and DIO vs DIO/Cu points at the $95 \%$ confidence levels. ${ }^{64} \mathrm{Cu}$ levels were monitored with a Fluke Biomedical CAL/RAD Mark IV Dose Calibrator and inductively coupled plasma-mass spectrometry (ICP-MS) was also performed. X-ray diffraction patterns were taken on a Siemens D-500 powder diffractometer with a Cu source. Room temperature magnetometry studies were performed on a Princeton Measurement Corporation vibrating 
sample magnetometer (VSM), while temperature dependent and cryogenic measurements were performed on

a Quantum Design superconducting quantum interference device (SQUID) magnetometer.

101

\section{Results and Discussion}

Hydrodynamic radius of the microwave-assisted synthesized nanoparticles were measured using DLS.

Using a maximum power of 300W $(n=3)$ a linear trend can be observed between particle size and increased

reaction time. Products were shown to increase in size by roughly $10 \mathrm{~nm}$ for every 5 minutes of reaction time as

microwave-assisted technique is able to reduce reaction times by providing uniform heating caused by an

instantaneous heating at the molecular level opposed to traditional conduction methods, such as refluxing.

Rapid and uniform heating allows for a more uniform and controllable size distribution.

Figure 1(b). All produced particles were within the optimal size range for in vivo testing $(\mathrm{d}<100 \mathrm{~nm})$, however, 
a.

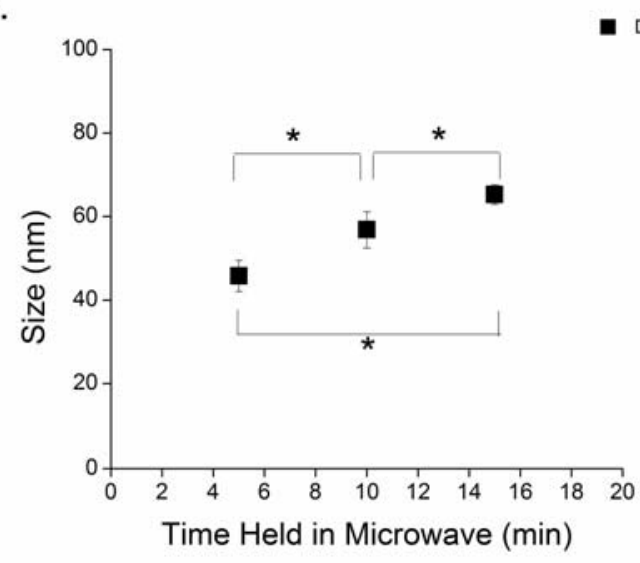

b.

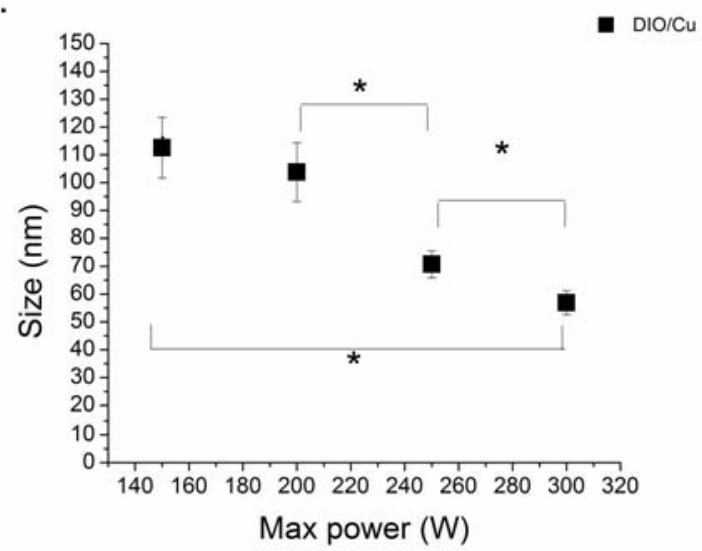

Figure 1. Dependence of particle size on duration of heating and max power. (a) Size increased with duration of heating time in the microwave while the max power was set to $300 \mathrm{~W}(\mathrm{DIO} / \mathrm{Cu}=\mathbf{a})(\mathrm{b})$ Size decreased when max power was increased and time was set to 10 minutes, as shown for ( $\mathrm{DIO} / \mathrm{Cu}=\mathbf{\square})$. $\left[{ }^{*}\right]$ designates the statistical significance between different trials where $p<0.05$

115
- DIO/Cu

icu 


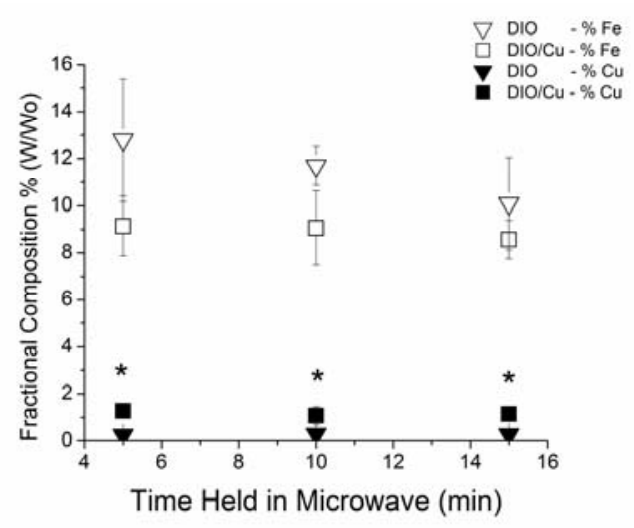

Figure 2. Iron and Copper Content for the Microwave Synthesized DIO and DIO/Cu. Percentage of iron

(Open) and copper (Closed) is shown where $\boldsymbol{\nabla}$ is representative of DIO, copper less reaction and $\boldsymbol{m}$ as $\mathrm{DIO} / \mathrm{Cu}$. Iron content in $\mathrm{DIO}$ can be seen to be slightly higher than that of DIO/Cu demonstrating that the incorporation of copper affects the iron content within the nanoparticle. Increase of copper levels seen in DIO/Cu compared to DIO was measured to be a $1 \%$ doping level of copper W/W\%. A significant change in iron content was not detected despite size increases over time signifying that the core composition remains the same leaving changes only in the shell size. $\left[{ }^{*}\right]$ designates the statistical significance between doped and undoped trials where $p<0.05$

Relaxometry was performed on the samples and is shown in Figure 3. For DIO/Cu there was no DIO and DIO/Cu were not significantly different between products from different reaction times. This confirms our earlier suggestion that the core particles form for only a short period of time and further suggests that the iron oxide cores within a larger particle are weakly interacting. However, relaxivities were significantly higher in the copper-doped DIO compared to the control DIO. In general the copper doped material was of smaller total size, contained significantly more copper, but iron content was not significantly different $(p<0.05)$. 
a.

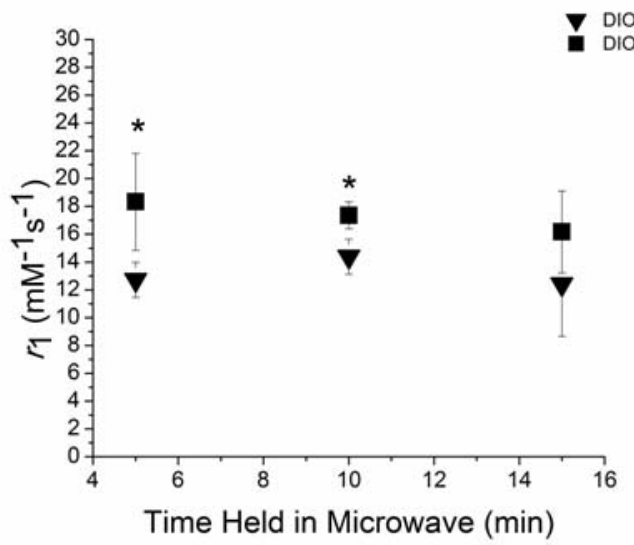

b.

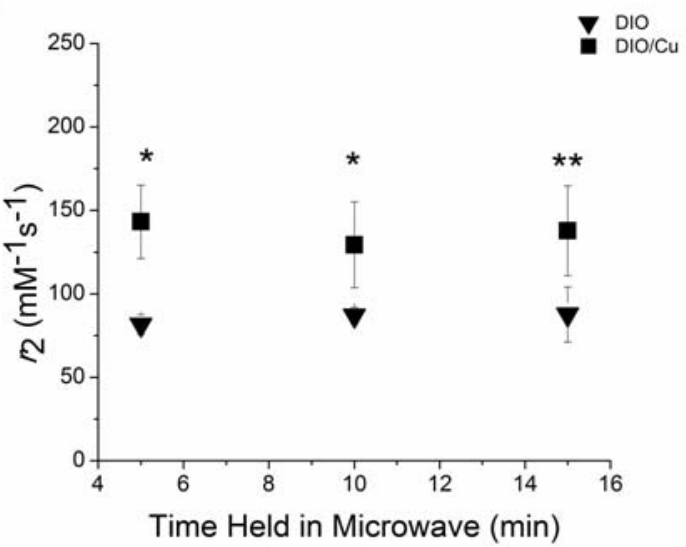

Figure 3. Relaxivity values for the microwave synthesized DIO and DIO/Cu. Relaxivity, $r_{1}(a)$ and $r_{2}$ (b). As anticipated, there was no significant difference between $r_{1}$ and $r_{2}$ values between different timed reactions due to equal iron concentrations within the nanoparticle products. However, a significant difference was seen between $\mathrm{DIO} / \mathrm{Cu}=\mathbf{m}, \mathrm{DIO}=\mathbf{\nabla} .\left[{ }^{*},{ }^{* *}\right]$ designates the statistical significance between different trials where $\left.{ }^{*} p<0.05,{ }^{*} p<0.10\right)$ AA, DLS and relaxivity results are summarized in Table 1; these data suggest that the increased particle 138 size that occurs under prolonged microwave reaction times is strictly due to increased thickness of surface 139 coating and accumulation of iron cores, not an increase in the size of the cores; this is evident by the lack of 140 change in iron content and relaxivity with synthesis time. The relaxivity values shown for DIO/Cu are 141 comparable to commercial agent such as Feridex ${ }^{\odot}$ and Resovist ${ }^{\odot}$ which have longitudinal relaxivity $\left(r_{1}\right)$ of 12.3 and $25 \mathrm{mM}^{-1} \mathrm{~s}^{-1}$ and transverse relaxivity $\left(r_{2}\right)$ of $191^{20}$ and $151^{21} \mathrm{mM}^{-1} \mathrm{~s}^{-1}$, respectively. 


\begin{tabular}{cccccccc} 
Type & $\begin{array}{c}\text { Time } \\
(\mathrm{min})\end{array}$ & $\begin{array}{c}\text { Total Size } \\
(\mathrm{nm})\end{array}$ & $\begin{array}{c}\text { Core Size } \\
(\mathrm{nm})\end{array}$ & $\mathrm{Fe}(\mathrm{mol})$ & $\mathrm{Cu}(\mathrm{mol})$ & $r_{1}\left(\mathrm{mM}^{-1} \mathrm{~s}^{-1}\right)$ & $r_{2}\left(\mathrm{mM}^{-1} \mathrm{~s}^{-1}\right)$ \\
\hline DIO/Cu & 5 & $45.7 \pm 3.7$ & $5.3 \pm 1.8$ & $1.6 \mathrm{E}^{-04} \pm 2.1 \mathrm{E}^{-05}$ & $1.3 \mathrm{E}^{-05} \pm 1.0 \mathrm{E}^{-05}$ & $17.1 \pm 2.3$ & $135.8 \pm 30.9$ \\
DIO & 5 & $61.9 \pm 12.6$ & $3.3 \pm 1.3$ & $1.1 \mathrm{E}^{-04} \pm 2.1 \mathrm{E}^{-05}$ & $1.8 \mathrm{E}^{-06} \pm 3.4 \mathrm{E}^{-07}$ & $15.1 \pm 2.3$ & $88.2 \pm 8.8$ \\
DIO/Cu & 10 & $56.8 \pm 4.3$ & $3.1 \pm 0.9$ & $1.2 \mathrm{E}^{-04} \pm 4.3 \mathrm{E}^{-05}$ & $7.7 \mathrm{E}^{-06} \pm 7.0 \mathrm{E}^{-06}$ & $15.8 \pm 3.4$ & $128.1 \pm 27.9$ \\
DIO & 10 & $63.9 \pm 5.8$ & $3.4 \pm 1.6$ & $9.6 \mathrm{E}^{-05} \pm 7.3 \mathrm{E}^{-06}$ & $2.1 \mathrm{E}^{-06} \pm 2.0 \mathrm{E}^{-07}$ & $14.9 \pm 3.8$ & $93.4 \pm 23.3$ \\
DIO/Cu & 15 & $65.3 \pm 2.4$ & $3.3 \pm 0.9$ & $1.5 \mathrm{E}^{-04} \pm 1.1 \mathrm{E}^{-05}$ & $9.6 \mathrm{E}^{-06} \pm 9.3 \mathrm{E}^{-06}$ & $15.5 \pm 1.5$ & $126.5 \pm 31.2$ \\
DIO & 15 & $71.8 \pm 16.6$ & $3.1 \pm 1.4$ & $9.6 \mathrm{E}^{-05} \pm 2.5 \mathrm{E}^{-05}$ & $2.2 \mathrm{E}^{-06} \pm 5.0 \mathrm{E}^{-07}$ & $11.6 \pm 4.9$ & $79.5 \pm 28.0$ \\
\hline
\end{tabular}

Table 1. Table comparing DIO and DIO/Cu trends for total size, core size, moles of iron, moles of copper, $r_{1}$ and $r_{2}$ values.

143

Magnetometry of DIO and DIO/Cu nanoparticles was performed; room temperature magnetic hysteresis loops are shown in Figure 4. Both the DIO and DIO/Cu were found to be superparamagnetic at room temperature, a necessity for application to $\mathrm{MRI}$, with a blocking temperature of $\sim 40 \mathrm{~K}$. From the blocking temperature we estimate the size of the doped and undoped iron oxide cores ${ }^{22}$ to be about $8 \mathrm{~nm}$. Saturation magnetization was measured at $10 \mathrm{~K}$ and found to be $16 \mathrm{emu} / \mathrm{g}$ and $12 \mathrm{emu} / \mathrm{g}$ for the $\mathrm{DIO}$ and $\mathrm{DIO} / \mathrm{Cu}$, respectively, normalized to the mass of the entire particle including the dextran coating. This reduction in saturation magnetization in $\mathrm{DIO} / \mathrm{Cu}$ is consistent with the inclusion of $\mathrm{Cu}$ in exchange for Fe. 


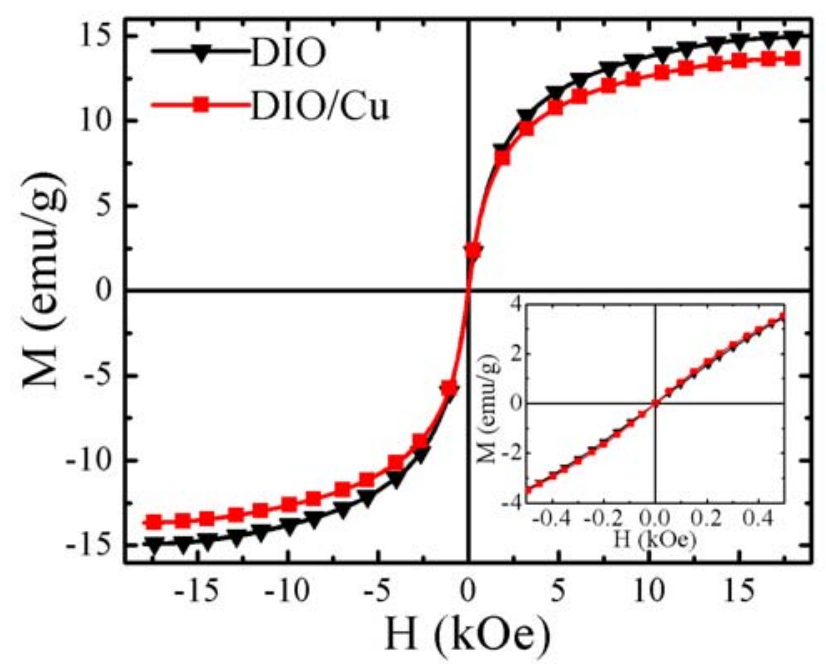

Figure 4. Hysteresis of DIO and Dio/Cu Nanoparticles. Room temperature magnetic

hysteresis loops of DIO and DIO/Cu nanoparticles. Inset shows a zoom-in view, confirming that these particles are superparamagnetic.

Dextran coating of iron oxide doped with copper allows for increased biocompatibility and blood retention

time. The addition of dextran also aids in size control; in the absence of dextran, bare iron oxide nanoparticles

tend to aggregate as shown in Figure 5(a) with a core size of $11.6 \pm 2.9 \mathrm{~nm}$. Iron oxide particles synthesized in the

observed for DIO/Cu. It can be noted that there were multiple $3 \mathrm{~nm}$ cores per $50 \mathrm{~nm}$ particles. The similarity in

core sizes indicates that the change in overall hydrodynamic size is due to thicker dextran coating.
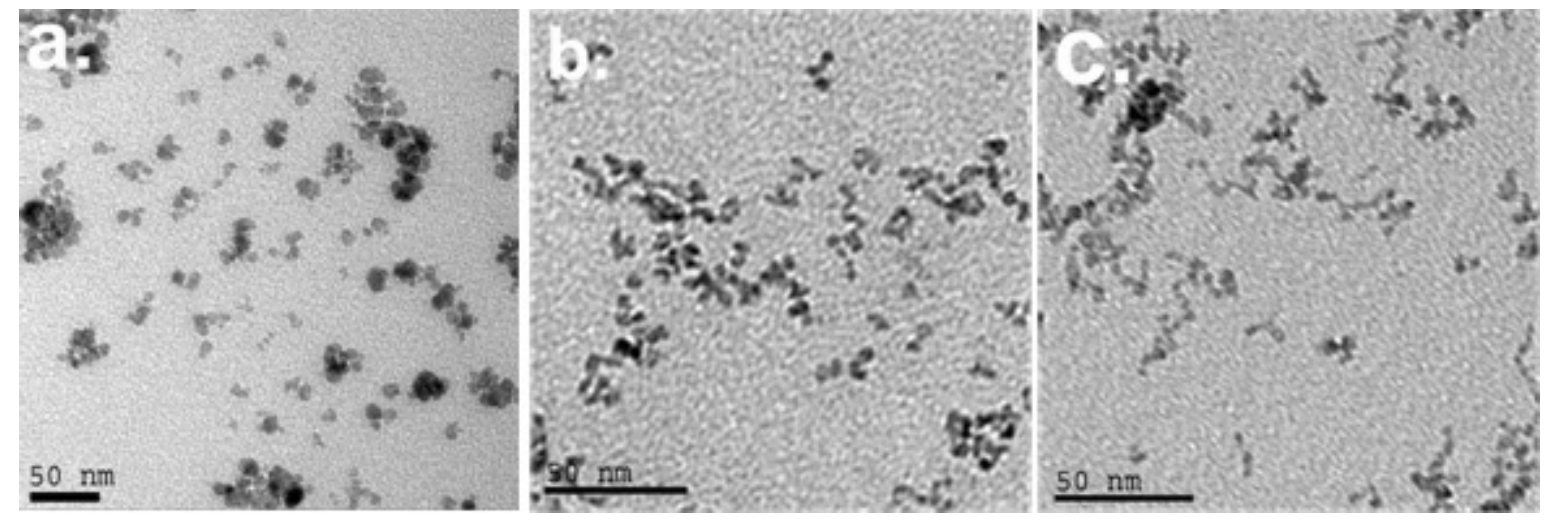

Figure 5. TEM of a. bare iron oxide, b. DIO and c. DIO/Cu Nanoparticles. Bare iron oxide prepared

using a similar protocol with no dextran present (a) shows iron oxide aggregating with increased core sizes. Nanoparticles prepared in the presence of dextran ( $B$ and $c$ ) are significantly more dispersed in nanoparticles containing copper and those without. 
160 (Figure 6a) include the C-O stretch at $1015 \mathrm{~cm}^{-1}, \mathrm{C}-\mathrm{H}$ bend at $1350 \mathrm{~cm}^{-1}, \mathrm{C}-\mathrm{H}$ stretch at $2900 \mathrm{~cm}^{-1}$ and O-H stretch 161 at $3350 \mathrm{~cm}^{-1}$. These same peaks are found for DIO (Figure $5 \mathrm{c}$ ) and DIO/Cu (Figure $5 \mathrm{~d}$ ) but not bare iron oxide 162 particles (Figure 6b). All of the particles showed peaks characteristic of hydroxyls, i.e. the O-H bend at $1404 \mathrm{~cm}^{-1}$ 163 and $\mathrm{O}-\mathrm{H}$ stretches at $3410 \mathrm{~cm}^{-1}$. The hydroxyl peaks observed may be attributed to water. In combination with 164 the increased size and dispersion illustrated by TEM in Figure 5, the FTIR results further confirm the coating of 165 dextran onto the DIO and DIO/Cu nanoparticles.

a.

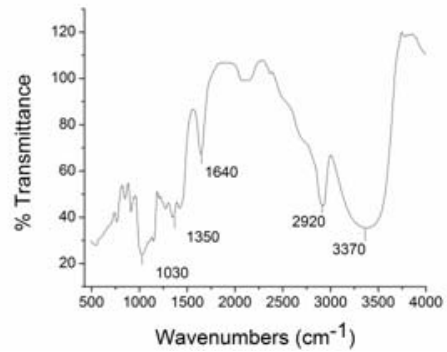

c.

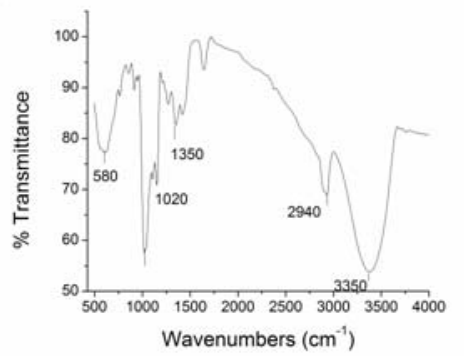

b.

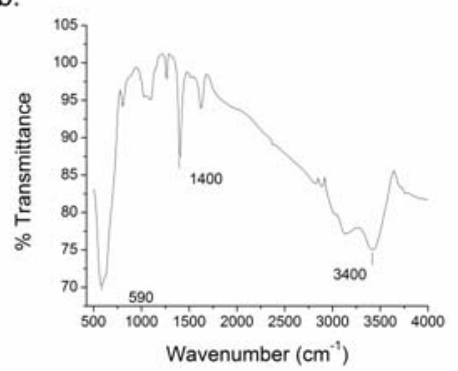

d.

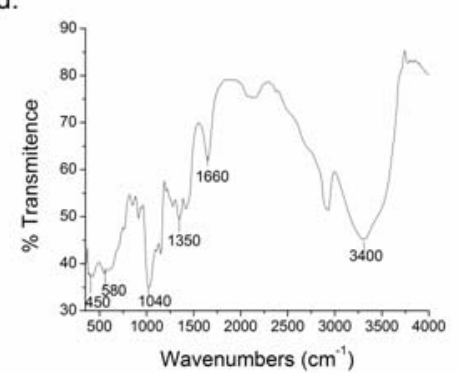

Figure 6. FTIR of a) dextran, b) bare iron oxide, c) DIO, D) DIO/Cu

Powder X-ray diffraction was performed to determine the structural effect of the incorporation of copper as well as the structure of iron oxide. Diffraction pattern for DIO is shown in Figure 7a and indicate the dominant phases are magnetite $\left(\mathrm{Fe}_{3} \mathrm{O}_{4}\right)$ and/or maghemite $\left(\gamma-\mathrm{Fe}_{2} \mathrm{O}_{3}\right)$; the presence of $\mathrm{Fe}^{2+}$ in the synthesis process does favor the magnetite phase. Copper added during copreciation is in the $2+$ oxidation state, working in direct competition with $\mathrm{Fe}^{2+}$ (from $\mathrm{FeCl}_{2}$ ) to occupy the octahedral site in the $\mathrm{Fe}_{3} \mathrm{O}_{4}$ structure, forming the cuprospinel structure. Due to the small amount of copper doping (10\%) the patterns for b) $\mathrm{DIO} / \mathrm{Cu}$, and c) $\mathrm{DIO} /{ }^{64} \mathrm{Cu}$ do not change signifigantly compared to a) undoped DIO. A small peak at $2 \theta=74.5^{\circ}$ in the $\mathrm{DIO} / \mathrm{Cu}$ sample suggests the formation of the cuprospinel structure, confirming the $\mathrm{Cu}^{2+}$ replacement of the $\mathrm{Fe}^{2+}$ in the original magnetite phase. 


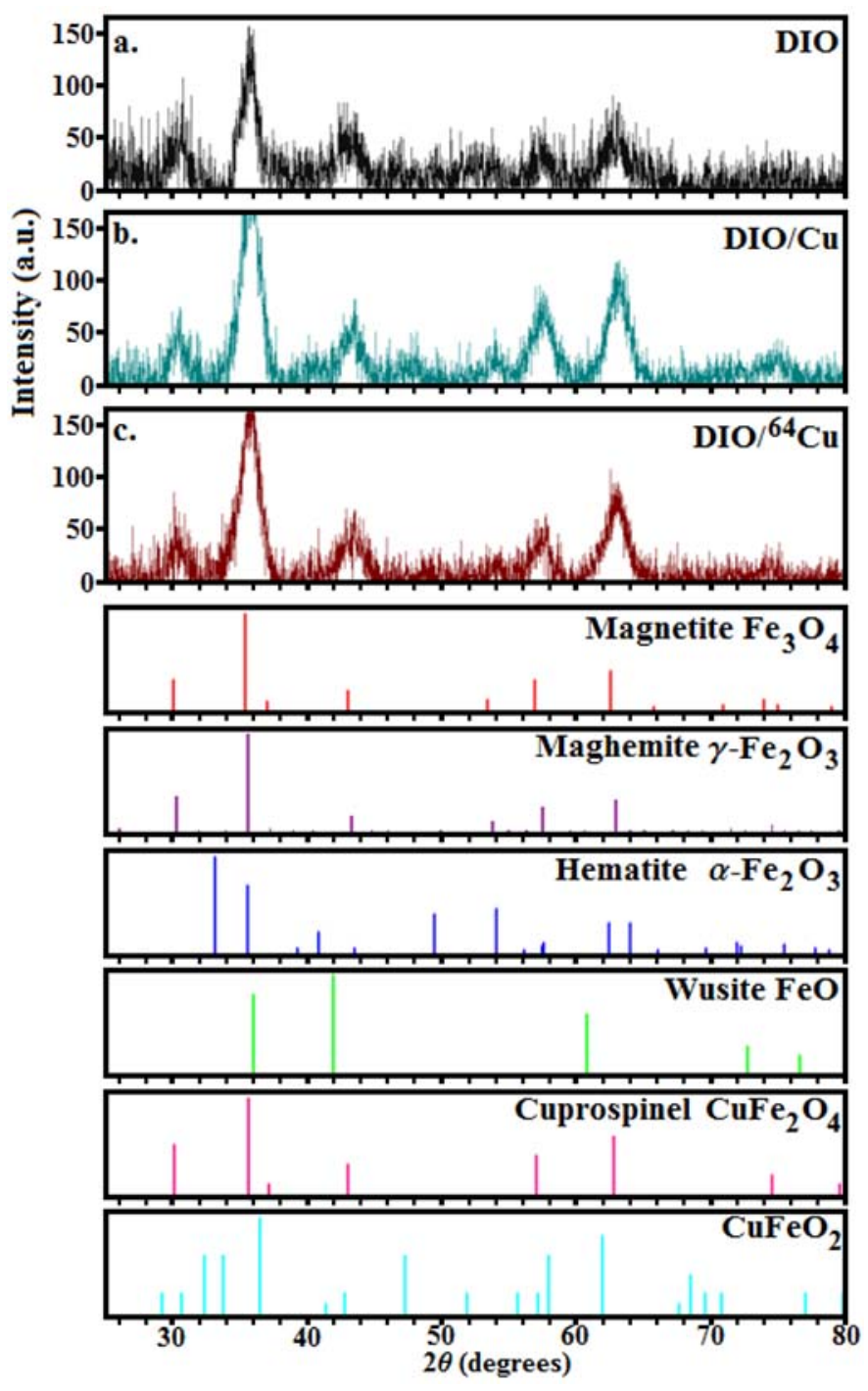

Figure 7. X-Ray Diffraction for Nanoparticles. X-ray diffraction patterns for (a) DIO, (b)

$\mathrm{DIO} / \mathrm{Cu}$, and (c) $\mathrm{DIO} /{ }^{64} \mathrm{Cu}$ and reference patterns for Magnetite, Maghemite, Hematite, Wusite,

\section{DIO/Cu-64 Synthesis}

Preliminary radiolabeling studies were performed to incorporate of copper-64 into DIO/Cu nanoparticles.

179 These experiments were performed on the same make and model of microwave used for the above studies but on a different machine that was approved for radioactive use. The primary differences for the instrument used

181 for radiolabeling were that it has a higher cooling air pressure and less temperature fluctuations. To control for instrument dependent artifacts $\mathrm{DIO}$, and $\mathrm{DIO} / \mathrm{Cu}-64$ were synthesized on this new instrument using a 1 minute 300W synthesis. As shown on Table 2, the hydrodynamic sizes were noted to be $36 \pm 2$, and $35 \pm 3 \mathrm{~nm}$ respectively as shown in supplemental data (S1). Trends demonstrating size increases with increased time were 
187 XRD pattern for the $\mathrm{DIO} /{ }^{64} \mathrm{Cu}$ is identical to $\mathrm{DIO} / \mathrm{Cu}$ and both are similar to DIO.

188

189

190

191

192

193

\begin{tabular}{cccc} 
Type & Total Size $(\mathrm{nm})$ & Core Size $(\mathrm{nm})$ & $r_{2}\left(\mathrm{mM}^{-1} \mathrm{~s}^{-1}\right)$ \\
\hline DIO & $35.6 \pm 1.5$, & $5.4 \pm 2.3$ & $121 \pm 14$ \\
DIO $/{ }^{64} \mathrm{Cu}$ & $35.3 \pm 3.3$ & $4.2 \pm 1.9$ & $100 \pm 20$
\end{tabular}

Table 2. Table comparing $\mathrm{DIO}$ and $\mathrm{DIO} /{ }^{64} \mathrm{Cu}$ trends for total size, core size and $r_{2}$ values for radio labeling conditions.

The presence of copper-64 was verified with gamma counting after purification to remove free, unincorporated copper. Initially $720 \pm 20 \mu \mathrm{Ci}$ of ${ }^{64} \mathrm{Cu}$ was added and after purification (with decay correction) $218 \pm 19 \mu \mathrm{Ci}$ remained, representing a $33 \%$ incorporation. The use of a high powered microwave for radiolabeling has a higher incorporation efficiency of Cu-64 compared to other previously mentioned radiolabeling methods ${ }^{7}$ as was observed for cold copper. ICP-MS was also performed and demonstrated that the elemental content in $\mathrm{DIO} /{ }^{64} \mathrm{Cu}$ for iron and copper were $1.1 \mathrm{E}^{-2} \mathrm{mg} / \mathrm{ml}$ and $9.7 \mathrm{E}^{-4} \mathrm{mg}$ respectively. This corresponds to roughly $4.9 \mathrm{E}^{-11} \pm 1.4 \mathrm{E}^{-12}$ moles of copper per sample. It is important to note that the sensitivity of PET is between nanomolar and picomolar levels making this probe well within the range of detectibility ${ }^{23}$. Furthermore, the starting amount of ${ }^{64} \mathrm{Cu}$ used for this preliminary study was far less than the amounts of cold copper used in the proof-of-concept studies $\left(5.3 \mathrm{E}^{-12} \pm 5.5 \mathrm{E}^{-13}\right.$ moles of ${ }^{64} \mathrm{Cu}$, compared to $1.5 \mathrm{E}^{-5}$ moles of cold copper). Higher ${ }^{64} \mathrm{Cu}$ content could be conceivably achieved by increasing the amount of ${ }^{64} \mathrm{Cu}$ initially included in the reaction.

\section{Conclusion}

In summary, we have demonstrated a microwave assisted synthesis technique to produce copperdoped and ${ }^{64} \mathrm{Cu}$-doped, iron oxide nanoparticles with a dextran coating. This novel synthesis allows for the rapid, size-controlled production of $\mathrm{DIO} / \mathrm{Cu}$, a novel contrast agent that is a proof-of-principle model for dual probe PET/MRI probes. Through the use of microwave heating, reaction times were significantly decreased from 120 minutes to 5 minutes while yielding similar sized particles. Size of the nanoparticles can be controlled simply by increasing the microwave heating time or by decreasing the maximum power. The rapid synthesis is greatly beneficial by leaving the radiolabel less time to decay. 
with diameters between 20 to $100 \mathrm{~nm}$, which is in a desirable size range. Size controlled synthesis of iron oxide + dextran particles is necessary due to the dependence of the magnetic states on the dispersion and aggregation, and the influence of size on the blood retention time. Nanoparticles over $80 \mathrm{~nm}$ are quickly removed by the reticuloendothelial system while nanoparticles smaller than $7-8 \mathrm{~nm}$ are removed by the kidneys, ${ }^{24,25}$ leaving a finite range in which nanoparticles have optimal blood retention time FTIR and TEM support that dextran is coated onto the iron oxide. The coating of dextran increases biocompatibility and allows for the ability for surface functionalization with specific targeting of biomarkers for interest as well as in vitro and in vivo studies.

\section{Acknowledgements}

223 This work has been supported by the Department of Energy (DOE DESC0002289) the National Science 224 Foundation (DMR-1008791). 
233 (1) Louie, A. Chemical Reviews. 2010, 110, 3146-95.

234 (2) Tsukamoto, E.; Ochi, S. Annual Nuclear Medicine. 2006, 20, 255-267.

235 (3) Cherry, S. R. Seminars in Nuclear Medicine. 2009, 39, 348-353.

236

237

(4) Slates, R. B.; Farahani, K.; Shao, Y.; Marsden, P. K.; Taylor, J.; Summers, P. E.; Williams, S.; Beech, J.; Cherry, S. R. Physics in Medicine and Biology. 1999, 44, 2015-27.

(5) Cho, S.; Jarrett, B. R.; Louie, A. Y.; Kauzlarich, S. M. Nanotechnology. 2006, 17, 640-644.

(6) Jarrett, B. R.; Frendo, M.; Vogan, J.; Louie, A. Y. Nanotechnology. 2007, 18, 15-18.

(7) Jarrett, B. R.; Gustafsson, B.; Kukis, D. L.; Louie, A. Y. Bioconjugate Chemistry. 2008, 19, 1496-504.

(8) Weizenecker, J.; Gleich, B.; Rahmer, J.; Dahnke, H.; Borgert, J. Physics in Medicine and Biology. 2009, 54, L1-L10.

(9) Dhenain, M. Magnetic Resonance Insights. 2008, 2, 75-91.

(10) Lee, H.; Li, Z.; Chen, K.; Hsu, A. R.; Xu, C.; Xie, J.; Sun, S.; Chen, X. Cell. 2008, 49, 1371-1379.

(11) Meares, C. In Recent Advances of Bioconjugation Chemistry in Molecular Imaging; Chen, X., Ed.; pp. $227-$ 244.

(12) Gedye, R.; Smith, F.; Westaway, K.; Ali, H.; Baldisera, L.; Laberge, L.; Rousell, J. Tetrahedron Letters. 1986, 27, 279-282.

249 (13) Smith, S. V. Journal of Inorganic Biochemistry. 2004, 98, 1874-901.

250 (14) Kooti, M.; Matturi, L. Int. Nano. Lett. 2011, 1, 38-42.

(16) Parsons, J. G.; Luna, C.; Botez, C. E.; Elizalde, J.; Gardea-Torresdey, J. L. The Journal of Physics and Chemistry of Solids. 2009, 70, 555-560.

254 (17) Deshmukh, R. G.; Badadhe, S. S.; Imtiaz, M. S. Materials Research Bulletin. 2009, 44, 1179-1182.

255 (18) Molday, R. S.; Mackenzie, D. Journal of Immunological Methods. 1982, 52, 353-367.

256 (19) Baker, M. Nature. 2010, 463, 977-980.

257 (20) Morcos, S. K. European radiology. 2010, 21, 496-500.

258 (21) Goyen, M.; Shamsi, K.; Schoenberg, S. O. European Radiology Supplements. 2006, 16, B9-B14. 
(22) Cho, S.; Shahin, A. M.; Long, G. J.; Davies, J. E.; Liu, K.; Grandjean, F.; Kauzlarich, S. M. Chem. Mater. 2006, 18, 960-967.

(23) Cheon, J.; Lee, J.-H. Accounts of chemical research. 2008, 41, 1630-40.

(24) Choi, H. S.; Liu, W.; Misra, P.; Tanaka, E.; Zimmer, J. P.; Itty Ipe, B.; Bawendi, M. G.; Frangioni, J. V. Nature biotechnology. 2007, 25, 1165-70.

(25) Welch, M. J.; Hawker, C. J.; Wooley, K. L. Journal of Nuclear medicine. 2009, 50, 1743-6.

(26) Kim, J.; Piao, Y.; Hyeon, T. Chemical Society Reviews. 2009, 372-390. 


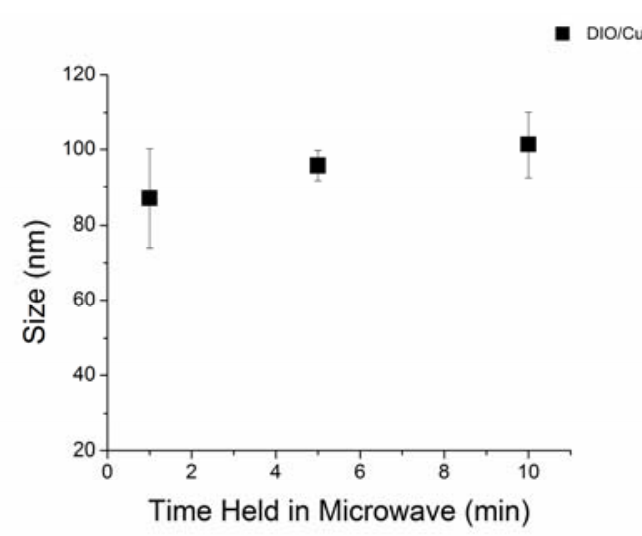

S1. DLS of Radiolabel conditions. DLS of 1 minute reactions at $85{ }^{\circ} \mathrm{C}$ with a max power of $300 \mathrm{~W}$ on a) $\mathrm{DIO}, \mathrm{b}) \mathrm{DIO} / \mathrm{Cu}, \mathrm{c}) \mathrm{DIO} / \mathrm{Cu}-64$. Average hydrodynamic sizes for particles were $35.6 \pm 1.5,59.6 \pm 14.19$, and $35.3 \pm 3.3 \mathrm{~nm}$ respectively with $P$ value $<0.1$ between all sets of data.

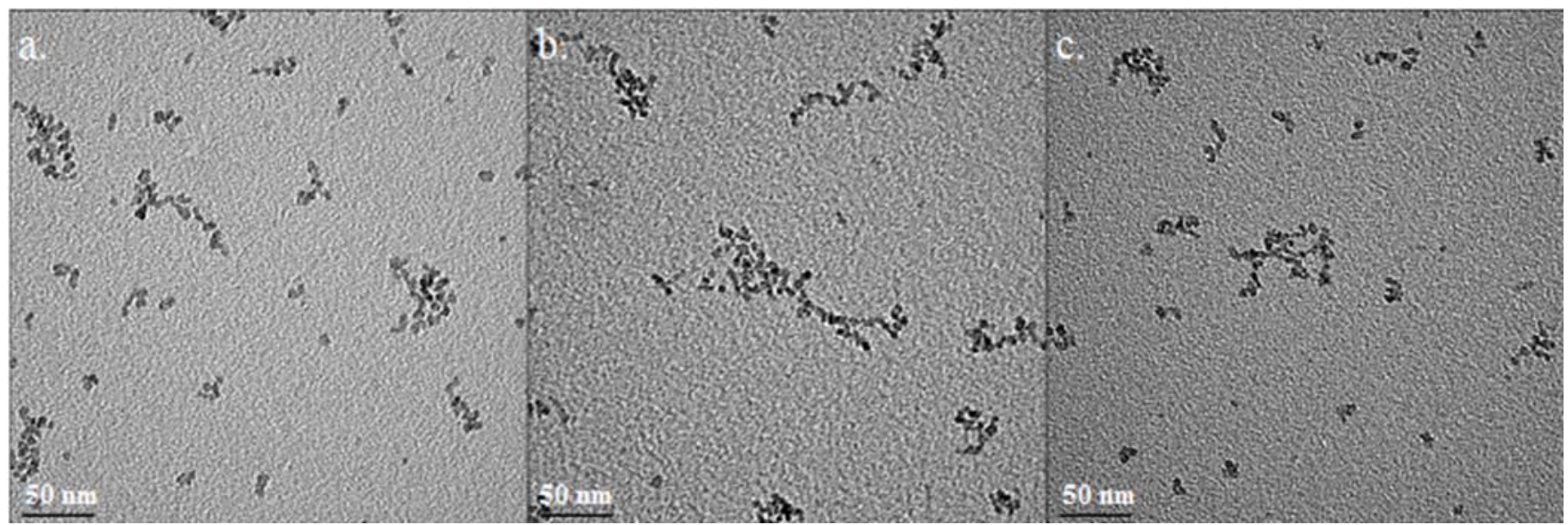

S2. TEM of Radiolabel Conditions. TEM of 1 minute reactions at $85^{\circ} \mathrm{C}$ with a max power of $300 \mathrm{~W}$ on a) DIO, b) DIO/Cu, c) DIO/Cu-64. With $n=500$, core sizes were noted to be a) $5.4 \pm 2.3 \mathrm{~nm}$, b) $4.6 \pm 1.7 \mathrm{~nm}, \mathrm{c}) 4.2 \pm$ $1.9 \mathrm{~nm}$. 